\title{
APPLICATION OF POLYNOMIAL TENSOR INTERPOLATION FOR TECHNICAL TESTS
}

\author{
Anita Ciekot $^{1}$, Grzegorz Biernat ${ }^{1}$, Tadeusz Fraczek ${ }^{2}$ \\ ${ }^{1}$ Institute of Mathematics, Czestochowa University of Technology \\ Czestochowa, Poland \\ ${ }^{2}$ Faculty of Production Engineering and Materials Technology, Czestochowa University of \\ Technology, Czestochowa, Poland \\ anita.ciekot@im.pcz.pl
}

Received: 7 January 2021; Accepted: 30 March 2021

\begin{abstract}
The main aim of this paper is a new formula of tensor interpolation by the polynomial of two variables. The formulas for interpolating polynomial coefficients are obtained using the Kronecker tensor product of matrices. The mathematical model for the diffusion process is presented. This paper is focused on determining the optimal parameters for this process by polynomial tensor interpolation of the obtained research results.
\end{abstract}

MSC 2010: 65D05, 41A05, $15 A 69$

Keywords: polynomial tensor interpolation, Kronecker product, diffusion process

\section{Introduction}

Generally, the interpolation process is a problem of constructing a function from a given set of data. The graph of this function has to pass through all the given points. This is the interpolation condition. The simplest method is interpolation by univariate polynomials, which is a known method in various fields of science including statistics, economics, optimization, control as well as practical problems [1-5]. However, polynomial interpolation in several variables is a rather new topic and quite complex, nevertheless, it is a method which can be applied to interpolate the obtained results of technical processes [4-6].

In this work, the interpolation formula by the polynomial of two variables is presented. The interpolating polynomial and its coefficients were formulated by means of the Kronecker tensor product. These formulas were given for the first time in papers [7-9].

This paper refers to applying polynomial tensor interpolation for the plasma nitriding process. This is one of the most frequently used methods to improve the mechanical properties of austenic steels from the group of chromium-nickel-molybdenum steels. This method simplifies the pretreatment of a steel surface before the nitriding process. The research results for polynomial tensor interpolation of the tribo- 
logical wear of austenitic 316L steel were analyzed for the first time in paper [4]. The obtained results of the nitrogen diffusion depth on austenitic steel type 316L according to AISI were interpolated by the polynomial of two variables - temperature and time.

This process was made for two various forms of locating the samples in a plasma chamber. The samples were located on the cathode, which is the first form, and the samples located on the cathode were additionally covered by an active screen which is the second form. The parameters were assumed for austenitic steels based on the preliminary analysis $[4,5]$.

This interpolation method of the particular results of the depth of nitrogen diffusion in the obtained surface layers will help to describe the properties of the considered nitriding process. Moreover, the obtained formula of the interpolating function gives the possibility to calculate the values of the depth of nitrogen in unmeasured points inside and outside the research zone.

\section{Kronecker tensor product of matrices}

The Kronecker product has its origin in group theory and has been successfully applied in various fields of science. Many interesting properties and theorems for the Kronecker product have been shown and proven in the literature on matrix analysis $[1,3,6]$. In this section, only a brief review of the properties of the Kronecker product of matrices will be presented.

\subsection{Definition of Kronecker product}

Let us consider two quadratic matrices $A=\left[a_{i j}\right]$ and $B=\left[b_{k l}\right]$ of degree $m$ and $n$ respectively. The tensor product of these matrices is defined as the block matrix

$$
A \otimes B=\left[a_{i j} B\right]=\left[\begin{array}{cccc}
a_{11} B & a_{12} B & \ldots & a_{1 m} B \\
a_{21} B & a_{22} B & \ldots & a_{2 m} B \\
\vdots & \vdots & \ddots & \vdots \\
a_{m 1} B & a_{m 2} B & \ldots & a_{m m} B
\end{array}\right]
$$

This tensor product is also known as the Kronecker product or the direct product. The Kronecker product for $k$ matrices is defined in [1] and [7].

\subsection{Properties of Kronecker product}

In this subsection a few basic properties of the Kronecker product are given. 
1. Matrices $A \otimes B$ and $B \otimes A$ are orthogonally similar.

This means that square matrix $U$ exists and $B \otimes A=U^{\text {transp }}(A \otimes B) U$

where $U^{\text {transp }} U=I$

2. $(\alpha A) \otimes(\beta B)=\alpha \beta(A \otimes B)$

3. $(A \otimes B)^{\text {transp }}=A^{\text {transp }} \otimes B^{\text {transp }}$

4. If $A$ and $B$ are invertible matrices then

$$
(A \otimes B)^{-1}=A^{-1} \otimes B^{-1}
$$

5. $\operatorname{det}(A \otimes B)=(\operatorname{det} A)^{n}(\operatorname{det} B)^{m}$

6. Element $(a \otimes b)_{r s}$ of matrix $A \otimes B$ is given by the product

$(a \otimes b)_{r s}=a_{i j} b_{k l}$

where

$r=(i-1) n+k$ and $s=(j-1) n+l$.

\subsection{Vandermonde matrix}

Let

$$
V_{p+1}=V_{p+1}\left(X_{0}, X_{1}, \ldots, X_{p}\right)=\left[\begin{array}{cccc}
1 & X_{0} & \ldots & X_{0}^{p} \\
1 & X_{1} & \ldots & X_{1}^{p} \\
\vdots & \vdots & \ddots & \vdots \\
1 & X_{p} & \ldots & X_{p}^{p}
\end{array}\right]
$$

be the Vandermonde matrix of degree $p+1$.

Proposition. The cofactors of matrix $V_{p+1}$ are given by the formula

$$
D_{i j}=(-1)^{i+j} \tau_{p-j}\left(X_{0}, \ldots, \hat{X}_{i}, \ldots, X_{p}\right) \operatorname{det} V_{p}\left(X_{0}, \ldots, \hat{X}_{i}, \ldots, X_{p}\right)
$$


where $\tau_{p-j}\left(X_{0}, \ldots, \hat{X}_{i}, \ldots, X_{p}\right)$ is the fundamental symmetric polynomial of rank $p-1$ of variables $X_{0}, \ldots, \hat{X}_{i}, \ldots, X_{p}, 0 \leq i, j \leq p$. Symbol $\hat{X}_{i}$ means omitting variable $X_{i}$. We assume $\tau_{0}=1$.

Proof. This property is easy to obtain on the one hand by extending the determinant

$$
\operatorname{det} V_{p+1}=\operatorname{det}\left[\begin{array}{cccc}
1 & X_{0} & \ldots & X_{0}^{p} \\
1 & X_{1} & \ldots & X_{1}^{p} \\
\vdots & \vdots & \ddots & \vdots \\
1 & X_{p} & \ldots & X_{p}^{p}
\end{array}\right]=\prod_{0 \leq k<l \leq p}\left(X_{l}-X_{k}\right)
$$

according to the $i$-th row, and on the other hand by sorting its value according to $X_{i}$ variable power.

Corollary. From formula (2) we obtained

$$
\frac{D_{i j}}{\operatorname{det} V_{p+1}}=\frac{(-1)^{i+j} \tau_{p-j}\left(X_{0}, \ldots, \hat{X}_{i}, \ldots, X_{p}\right)}{\pi_{i}}, \quad 0 \leq i, j \leq p
$$

where $\pi_{i}=\left(X_{p}-X_{i}\right) \cdot \ldots \cdot\left(X_{i+1}-X_{i}\right)\left(X_{i}-X_{i-1}\right) \ldots\left(X_{i}-X_{0}\right)$.

\section{Polynomial tensor interpolation}

The coefficients matrix $\left[c_{i k}\right]$ of the polynomial interpolation

$$
W(X, Y)=\sum_{0 \leq i \leq p, 0 \leq k \leq q} c_{i k} X^{i} Y^{k}
$$

is unknown. The result matrix $\left[w_{j l}\right]=\left[W\left(X_{j}, Y_{l}\right)\right], 0 \leq j \leq p, 0 \leq l \leq q$, on nodes $\left(X_{j}, Y_{l}\right)$ is known.

The coefficients of the polynomial are determined from the linear system of equations

$$
\left(\left[X_{i}^{j}\right] \otimes\left[Y_{k}^{l}\right]\right) \operatorname{vec}\left[c_{i k}\right]=\operatorname{vec}\left[w_{j l}\right]
$$

where the nodes matrix is the Kronecker product of the Vandermonde matrices $\left[X_{i}^{j}\right]$, $\left[Y_{k}^{l}\right]$ and the operator "vec" rearrange the elements of the coefficient matrix $\left[c_{i k}\right]$ 
and the results matrix $\left[w_{j l}\right]$ in the form of a column vectors. This means that we select ordering

$$
00,01, \ldots . ., 0 q, 10,11, . .1 q, \ldots, p 0, p 1, \ldots . . p q
$$

with the sequence shown above.

Then the searching coefficients column has the form

$$
\operatorname{vec}\left[c_{i k}\right]=\frac{1}{\operatorname{det}\left[X_{i}^{j}\right]} \frac{1}{\operatorname{det}\left[Y_{k}^{l}\right]}\left(D_{\left[X_{i}^{j}\right]} \otimes D_{\left[Y_{k}^{l}\right]}\right)^{\text {transp }} \operatorname{vec}\left[w_{j l}\right]
$$

According to property 6 we, obtain the formula for the coefficients

$$
c_{i k}=\sum_{0 \leq j \leq p, 0 \leq l \leq q} w_{j l} \frac{\left(D_{\left[X_{i}^{j}\right]}\right)_{i j}}{\operatorname{det}\left[X_{i}^{j}\right]} \frac{\left(D_{\left[Y_{k}^{l}\right]}\right)_{k l}}{\operatorname{det}\left[Y_{k}^{l}\right]}
$$

where $D_{\left[X_{i}^{j}\right]}, D_{\left[Y_{k}^{l}\right]}$ are the cofactors of the matrices $\left[X_{i}^{j}\right],\left[Y_{k}^{l}\right]$ and because the fractions shown above have the known form (3) then:

$c_{i k}=(-1)^{i+k} \sum_{0 \leq j \leq p, 0 \leq l \leq q}(-1)^{j+l} w_{j l} \frac{\tau_{p-i}\left(X_{0}, \ldots \hat{X}_{j}, \ldots, X_{p-1}\right)}{\pi_{1 j}} \ldots \frac{\tau_{q-k}\left(Y_{0}, \ldots \hat{Y}_{l}, \ldots, Y_{q-1}\right)}{\pi_{2 l}}$

where

$$
\begin{aligned}
& \pi_{1 j}=\left(X_{p}-X_{j}\right) \cdot \ldots \cdot\left(X_{j+1}-X_{j}\right)\left(X_{j}-X_{j-1}\right) \cdot \ldots \cdot\left(X_{j}-X_{0}\right) \\
& \pi_{2 l}=\left(Y_{q}-Y_{l}\right) \cdot \ldots \cdot\left(Y_{l+1}-Y_{l}\right)\left(Y_{l}-Y_{l-1}\right) \cdot \ldots \cdot\left(Y_{l}-Y_{0}\right)
\end{aligned}
$$

\section{Polynomial tensor interpolation - exemplary numerical results}

The numerical results have been calculated for one of the most frequently used austenitic steels type 316L according to AISI. The plasma nitriding processes were prepared according to the rules of mathematical research planning. The researchers were using the equipment for plasma treatment with a JON-600 cooling anode. The measurements were performed in hydrogen-nitrogen plasma for the following nitriding parameters: process pressure $p=150 \mathrm{~Pa}$, temperature range $T=325 \div 400^{\circ} \mathrm{C}$, and process time $t=2 \div 4 \mathrm{~h}$. 
Two forms of locating the samples in the plasma chamber were applied:

- the samples were located directly on the cathode,

- the samples located on the cathode were additionally covered by an active screen (made by a perforated austenitic steel sheet).

The depth of diffusion was described by the interpolating polynomial of two variables that characterized the nitriding process: temperature $T$ and time $t$ [5]. The following parameters were assumed to obtain the depth of diffusion: $T_{1}=325^{\circ} \mathrm{C}$, $T_{2}=350^{\circ} \mathrm{C}, T_{3}=375^{\circ} \mathrm{C}, T_{4}=400^{\circ} \mathrm{C}$ and $t_{1}=2 \mathrm{~h}, t_{2}=3 \mathrm{~h}, t_{3}=4 \mathrm{~h}$.

During the experimental plasma nitriding process, the following values of diffusion depth were obtained:

- for the active screen method

$$
\left[\left(w_{s}\right)_{j l}\right]=[1.07 ; 1.78 ; 2.61 ; 2.45 ; 3.28 ; 3.68 ; 3.69 ; 3.81 ; 4.01 ; 4.62 ; 5.02 ; 6.34]^{\text {transp }}
$$

- for cathode nitriding

$$
\left[\left(w_{c}\right)_{j l}\right]=[0.56 ; 0.98 ; 1.01 ; 0.88 ; 1.38 ; 1.80 ; 1.44 ; 1.96 ; 2.15 ; 1.61 ; 2.26 ; 2.51]^{\text {transp }}
$$

In the case of plasma nitriding using the active screen method, the interpolating polynomial is expressed by the following formula:

$$
W_{s}(T, t)=\sum_{\substack{1 \leq i \leq 4 \\ 1 \leq k \leq 3}}\left(c_{s}\right)_{i k} T^{i-1} t^{k-1}
$$

with the coefficients

$$
\left(c_{s}\right)_{i k}=(-1)^{i+k} \sum_{\substack{1 \leq j \leq 4 \\ 1 \leq l \leq 3}}(-1)^{j+l}\left(w_{s}\right)_{j l} \frac{\tau_{4-i}\left(T_{1}, \ldots \hat{T}_{j}, \ldots, T_{4}\right)}{\pi_{1 j}} \ldots \frac{\tau_{3-k}\left(t_{1}, \ldots \hat{t}_{l}, \ldots, t_{3}\right)}{\pi_{2 l}}
$$

and for cathode nitriding

$$
W_{c}(T, t)=\sum_{\substack{1 \leq i \leq 4 \\ 1 \leq k \leq 3}}\left(c_{c}\right)_{i k} T^{i-1} t^{k-1}
$$

with the coefficients

$$
\left(c_{c}\right)_{i k}=(-1)^{i+k} \sum_{\substack{1 \leq j \leq 4 \\ 1 \leq l \leq 3}}(-1)^{j+l}\left(w_{c}\right)_{j l} \frac{\tau_{4-i}\left(T_{1}, \ldots \hat{T}_{j}, \ldots, T_{4}\right)}{\pi_{1 j}} \ldots \frac{\tau_{3-k}\left(t_{1}, \ldots \hat{t}_{l}, \ldots, t_{3}\right)}{\pi_{2 l}}
$$

where 


$$
\begin{array}{ll}
\pi_{11}=\left(T_{4}-T_{1}\right)\left(T_{3}-T_{1}\right)\left(T_{2}-T_{1}\right) & \pi_{21}=\left(t_{3}-t_{1}\right)\left(t_{2}-t_{1}\right) \\
\pi_{12}=\left(T_{4}-T_{2}\right)\left(T_{3}-T_{2}\right)\left(T_{2}-T_{1}\right) & \pi_{22}=\left(t_{3}-t_{2}\right)\left(t_{2}-t_{1}\right) \\
\pi_{13}=\left(T_{4}-T_{3}\right)\left(T_{3}-T_{2}\right)\left(T_{3}-T_{1}\right) & \pi_{23}=\left(t_{3}-t_{2}\right)\left(t_{3}-t_{1}\right) \\
\pi_{14}=\left(T_{4}-T_{3}\right)\left(T_{4}-T_{2}\right)\left(T_{4}-T_{1}\right) &
\end{array}
$$

Here, $\left[T_{i}^{j}\right]$ describes the temperatures of the nitriding process, and $\left[t_{k}^{l}\right]$ times the exposure of a specimen at a given temperature.

The computations were performed by means of Maple software. Figure 1 shows the polynomial interpolation of obtained results of nitrogen diffusion depth as a result of plasma nitriding the using active screen method and cathode plasma nitriding. The experimental data are represented by dots.

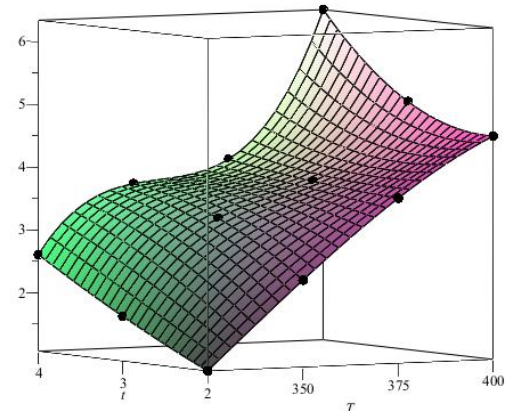

a)

Fig. 1. Polynomial interpolation of obtained results of nitrogen diffusion depth as a result of: a) plasma nitriding using the active screen method and $b$ ) cathode plasma nitriding

\section{Conclusions}

In this article, the effective formulas for the coefficients of the interpolation polynomial were derived by use of the Kronecker tensor product. It is a new point of view in interpolation theory. The presented method was applied to interpolate the results obtained in the nitriding process. The polynomial of two variables: temperature and time was applied to obtain the interpolating function. The proposed formulas can be extended to a larger number of parameters (for example: pressure) but this requires increasing the number of measurements in the interpolation nodes. Furthermore, the number of nodes could also increase, and this would improve the degree of accuracy of the interpolation. 


\section{References}

[1] Graham, A. (1981). Kronecker Products and Matrix Calculus with Applications. Ellis Horwood Ltd.

[2] Kincaid, D., \& Chnej, W. (2002). Numerical Analysis, Mathematics of Scientific Computing. The University of Texas at Austin.

[3] Biernat, G., \& Ciekot, A. (2009). The polynomial tensor interpolation. Arithmetical case. Scientific Research of the Institute of Mathematics and Computer Science Czestochowa University of Technology, 1(8), 7-11.

[4] Frączek, T., Olejnik, M., Knapinski, M., \& Biernat, G. (2010). Tensor interpolation of tribological wear in ionnitriding of 316L steel. Solid State Phenomena, 165, 43-49.

[5] Olejnik, M. (2011). Niskotemperaturowe $i$ krótkookresowe azotowanie jarzeniowe stali austenicznej X2CrNiMo 17012-2, PhD Thesis, Czestochowa (in Polish).

[6] Biernat, G., \& Ciekot, A. (2007). The polynomial interpolation for technical experiments. Scientific Research of the Institute of Mathematics and Computer Science Czestochowa University of Technology, 1(6), 19-22.

[7] Gasca, M., \& Sauer, T. (2000). On the history of multivariate polynomial interpolation. Journal of Computational and Applied Mathematics, 122, 23-35.

[8] Thomas, D.H. (1976). A natural tensor product interpolation formula and pseudoinverse of a matrix. Linear Algebra and Its Applications, 13, 239-250.

[9] Kilicman, A., \& Al Zhour, Z.A.A. (2007). Kronecker operational matrices for fractional calculus and some applications. Applied Mathematics and Computation, 187, 250-265. 